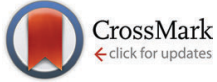

Cite this: Phys. Chem. Chem. Phys., 2016, 18, 28370

Received 22nd June 2016

Accepted 19th September 2016

DOI: $10.1039 / c 6 c p 04372 k$

www.rsc.org/pccp

\title{
Raman excess spectroscopy vs. principal component analysis: probing the intermolecular interactions between chiral molecules and imidazolium-based ionic liquids
}

\author{
H. Koch, K. Noack and S. Will*
}

\begin{abstract}
Raman spectroscopy is a very sensitive and specific measurement tool for probing intermolecular interaction structures. As imidazolium-based ionic liquids can favorably be used for enantioseparation, in this work two approaches for Raman signal analysis, namely by means of excess spectra and principal component analysis (PCA), are compared to extract detailed information about the interaction structure of the enantiomers D- and L-glucose in an aqueous solution of the ionic liquid [EMIM] $\left[\mathrm{EtSO}_{4}\right]$. In contrast to the excess calculations, the loadings obtained from PCA lead to significant results since the interactions are weighted by their strength and significance. Moreover, the analysis of the weighted vibrations in the loadings indicate that hydrogen bonds are particularly formed between the ethyl sulphate anion of [EMIM] [EtSO 4 and the hydrogen atom of the $\mathrm{OH}$-group at the $\mathrm{C}_{6}$-atom of glucose.
\end{abstract}

\section{Introduction}

Enantiomers are chiral molecules with an identical chemical formula, but opposite configuration. They are characterized by the same physicochemical properties, but different optical activity, e.g. their ability to rotate linearly polarized radiation. Moreover, $60-70 \%$ of all sold drugs are chiral molecules and that with an increasing demand. ${ }^{1}$ However, since enantiomers can have different physiological effects, it is of utmost importance to ensure enantiopurity. A tragic example of both enantiomers being bioactive is thalidomide: D-thalidomide acts as a sedative, whereas L-thalidomide is teratogenic. ${ }^{2-4}$ Enantioseparation, however, is difficult as both enantiomers possess identical physicochemical properties. Moreover, by chemical synthesis usually both enantiomers are formed in equal amounts yielding a 1:1 mixture termed racemate. To separate the enantiomers, today the most common techniques are chromatography, crystallization, ${ }^{5-8}$ enzymatic resolution, ${ }^{5-7}$ diastereomer separation, membrane processes ${ }^{6,8}$ or a combination of these techniques. $^{6}$ To investigate the often underlying enantioselective interactions and use them for process design, optical measurement techniques can favourably be employed. In general, spectroscopic methods, like optical rotary dispersion (ORD),, 10 circular dichroism (CD), ${ }^{9-11}$ and Raman optical activity (ROA), ${ }^{12-15}$

Lehrstuhl für Technische Thermodynamik (LTT) and Erlangen Graduate School in Advanced Optical Technologies (SAOT), Friedrich-Alexander-Universität Erlangen-Nürnberg, Am Weichselgarten 8, 91058 Erlangen, Germany.

E-mail: stefan.will@fau.de are used to distinguish the enantiomers of chiral molecules. Wang et al. (2014) presented surface enhanced Raman spectroscopy (SERS) to study enantioselective interactions. ${ }^{16}$ However, SERS is an expensive measurement technique. In contrast to the wide held belief that Raman spectroscopy cannot be used to optically separate two enantiomers, two very recent studies presented a Raman approach which proves that Raman spectroscopy is a very promising and powerful tool for discriminating enantiomers. ${ }^{17,18}$ Furthermore, Raman signals are highly sensitive and selective, and thus even slight changes in a molecular system can be uncovered. Thus, in the work presented here, we want to investigate the enantioselective interactions between the enantiomers of glucose and the co-solvent 1-ethyl-3-methylimidazolium ethyl sulphate [EMIM] $\left[\mathrm{EtSO}_{4}\right]$, which is an ionic liquid. Room temperature ionic liquids (RTILs) belong to a group of organic salts with melting points near room temperature or by convention below $100{ }^{\circ} \mathrm{C} .{ }^{19}$ RTILs are frequently used as alternative solvents, e.g. in the field of separation science as extraction solvents. ${ }^{20-24}$ Since an improved chiral separation was recognized by using achiral imidazolium-based ionic liquids, ${ }^{25,26}$ [EMIM] [EtSO $\left.{ }_{4}\right]$ was chosen as a co-solvent for this work. In addition, it is a very well studied RTIL. ${ }^{27-34}$ In this paper we discuss how information about intermolecular interactions can be extracted by the evaluation of Raman excess signals and from principal component analysis (PCA) ${ }^{35-43}$ to discriminate the enantiomers of glucose. The ultimate goal of this work is to provide a means for the in situ characterisation of enantiomers using Raman spectroscopy for aiding enantioseparation. 


\section{Experimental}

D- and L-glucose (purity: 98\%) (Fig. 1) were purchased from VWR (Germany). 1-Ethyl-3-methylimidazolium ethyl sulphate, [EMIM] $\left[\mathrm{EtSO}_{4}\right]$ (Fig. 2) (purity: 99\%), was purchased from Merck KGA (Germany). Quaternary mixtures of D-, L-glucose, [EMIM] $\left[\mathrm{EtSO}_{4}\right]$ and water were prepared with $r_{\mathrm{m}}=0.32,0.43$, $0.46,0.66,0.80,1.00,1.22,1.50,2.18,2.50$ and $3.04( \pm 0.001)$, where $r_{\mathrm{m}}$ represents the $\mathrm{D}$ - to L-ratio,

$$
r_{\mathrm{m}}=\frac{m_{\mathrm{D}}}{m_{\mathrm{L}}}
$$

with $m_{\mathrm{D}}$ being the weighted mass of D-glucose and $m_{\mathrm{L}}$ that of L-glucose. The mass fraction of [EMIM] $\left[\mathrm{EtSO}_{4}\right]$ was about $50 \mathrm{wt} \%$ in every solution.

The experiments were accomplished by means of a Raman set-up using a diode laser at $785 \mathrm{~nm}$ (Toptica, $P=100 \mathrm{~mW}$, bandwidth: $0.12 \mathrm{~nm}$ ) for excitation and polarization-resolved detection. The Raman signals were collected by detection optics in a $90^{\circ}$ arrangement in a quartz cuvette with a quadratic cross section (edge length: $1 \mathrm{~cm}$ ), which was filled with $1 \mathrm{ml}$ of the sample solution (scattering volume: $c a .55 \mathrm{~mm}^{3}$ ). A notch filter (CW: $785 \pm 2 \mathrm{~nm}$, FWHM: $33 \mathrm{~nm}$ ) and two long-pass filters (790 $\mathrm{nm}$ and $800 \mathrm{~nm}$ cut-off) were used to block the elastically scattered light. A polarizing beam splitter divided the Raman signal into its vertical and horizontal signal components. The polarized signals were each focused at a time into an optical fibre coupled to a CCD spectrometer (Ocean Optics, QE65000) with a resolution of about $4 \mathrm{~cm}^{-1}$. The signal intensities of the vertical and the horizontal signals were added. As the set-up allows for a polarization-resolved detection, we first checked whether a polarization-evaluation would lead to even more significant results. As this was not the case, the two components were simply added. The pre-processing also included a normalization of the spectra.

For a conventional excess model for investigating intermolecular interactions, the Raman excess spectra, $R_{\text {excess }}$, were calculated from the difference between experimental and ideal Raman signals, respectively:

$$
R_{\text {excess }}=R_{\text {exp }}-R_{\text {ideal }}
$$

The ideal spectra were determined by summing up the aqueous $\mathrm{D}-\left(R_{\mathrm{D}}^{0}\right)$ and $\mathrm{L}-\left(R_{\mathrm{L}}^{0}\right)$ enantiopure spectra, pure water $\left(R_{\mathrm{water}}^{0}\right)$ and $[\mathrm{EMIM}]\left[\mathrm{EtSO}_{4}\right]$ spectra $\left(R_{\left[\mathrm{EMIM}^{0}\left[\mathrm{EtSO}_{4}\right]\right.}^{0}\right)$. In addition, each spectrum was weighted by a factor $x$ according to the mass fraction of the respective component in the mixture:

$$
R_{\text {ideal }}=x_{\mathrm{D}} R_{\mathrm{D}}^{0}+x_{\mathrm{L}} R_{\mathrm{L}}^{0}+x_{\text {water }} R_{\text {water }}^{0}+x_{[\mathrm{EMIM}]\left[\mathrm{EtSO}_{4}\right]}
$$
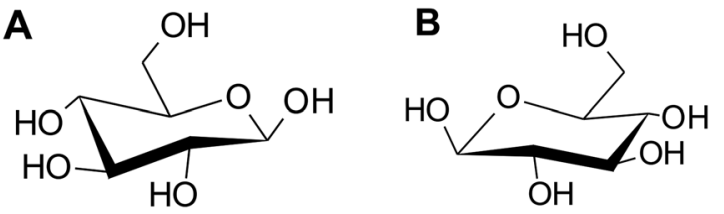

Fig. 1 Structural formulae of $D-(A)$ and L-glucose (B).<smiles>CCn1ccn(C)[cH+]1</smiles><smiles>CCOS(=O)(=O)[O-]</smiles>

Fig. 2 Structural formula of [EMIM] [EtSO 4 ]

For the ideal spectra the Raman spectra with 6, 16 and $32 \mathrm{wt} \%$ of D- or L-glucose, respectively, dissolved in water were used.

Furthermore, to receive information about the D-L-glucose interactions a weighted spectrum of [EMIM] $\left[\mathrm{EtSO}_{4}\right]$ and water was subtracted. The weighting is based on the division of the net peak intensities at $772 \mathrm{~cm}^{-1}$ since this peak reflects the $\delta\left(\mathrm{CH}, \mathrm{CH}_{2}, \mathrm{SO}\right)$-vibration of [EMIM] $\left[\mathrm{EtSO}_{4}\right]$ and thus represents both the anion and cation of the RTIL:

$$
x=\frac{\text { net peak intensity }}{\text { quaternary mixture }}
$$

By subtracting this spectrum, the RTIL-water interactions are discounted. Hence, the peaks in the excess spectra can be assigned to RTIL-D- or L-glucose interactions as well as to D-L-glucose interactions.

For comparison, Raman spectra were analysed by means of principal component analysis (PCA) which is a linear transformation method and extracts relevant information from experimental data sets by reducing the dimensionality. ${ }^{38,44,45}$ This is accomplished by calculating the principal components (PCs) or the loadings of the experimental data using the Matlab code $p c a$ (Matlab R2013b). The PCs are a set of linearly uncorrelated variables being orthogonal to each other. Thus, the PCs represent the directions of maximal signal variance in descending order. The most relevant PCs serve as a new coordinate system onto which the original data are projected, and, therefore, reduced in dimensionality. The results of the PCA were used to select those molecule parts or bonds that are most significantly influenced by changes in the intra- and intermolecular interaction structure.

\section{Results and discussion}

The extraction of qualitative information about inter- and intramolecular interactions in multicomponent mixtures can be performed by applying excess spectroscopy. ${ }^{33}$ However, when dealing with different phases at room temperature, solvation effects result in further excess which has to be considered for calculating the ideal spectra. As an example, for determining the excess Raman spectrum of a quaternary mixture of $\mathrm{D}$ - and L-glucose, water and [EMIM] [EtSO $\left.{ }_{4}\right]$ the ideal mixture spectrum has to include a measure for the interactions between the single glucose molecules and water since glucose is solid at room temperature. This effect is presented in Fig. 3, which shows the 


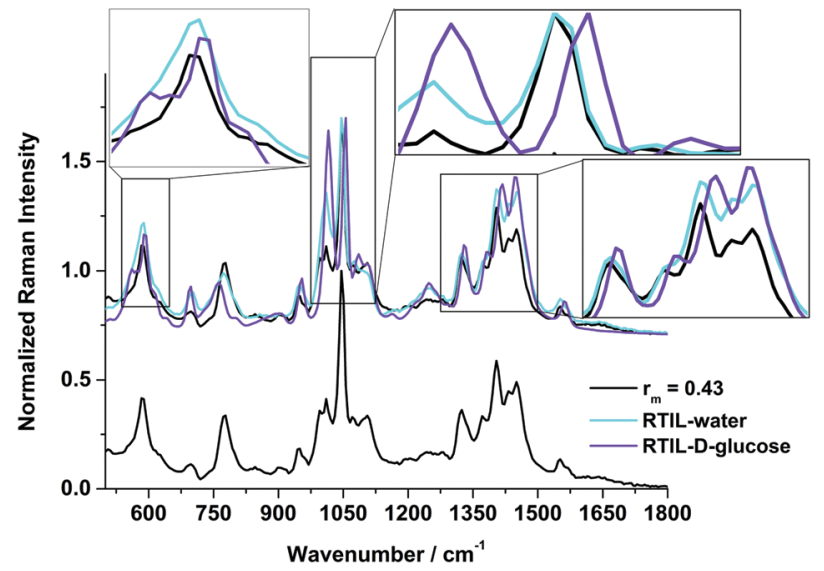

Fig. 3 Comparison of the Raman excess spectrum of the quaternary mixture with $r_{\mathrm{m}}=0.43$ and the binary $[\mathrm{EMIM}]\left[\mathrm{EtSO}_{4}\right] /$ water and [EMIM] [EtSO 4 ]/D-glucose mixtures.

Raman excess spectra of the RTIL/water and RTIL/D-glucose binary mixtures as well as that of the quaternary mixture of $\mathrm{D}^{-}$, L-glucose, water and [EMIM] $\left[\mathrm{EtSO}_{4}\right]$ with $r_{\mathrm{m}}=0.43$. By comparing the excess spectrum with the two Raman spectra of the binary mixtures of the RTIL and water or D-glucose it is possible to extract information about the intermolecular interactions in the excess spectrum. This is due to the fact that red or blue shifts occur when the RTIL interacts with water or glucose, respectively. Hence, these shifts can be used to extract information about interactions occurring in the quaternary mixture.

The insets in Fig. 3 point out some interaction changes. This comparison results in the assumption that intermolecular interactions between the RTIL and water or glucose occur with a similar strength since both of them get obvious with nearly the same number of peak-shifts. The comparison of these spectra features blue or red shifts, respectively, of the excess spectrum, which enable the assignment of the occurring interactions in the quaternary mixture.

To evaluate this finding, the study of Carneiro et al. $(2012)^{46}$ is considered, which shows that the ethyl sulphate anion has a strong affinity for forming hydrogen bonds with the hydrogen atoms of glucose. Moreover, the study of Molteni and Parrinello $(1998)^{47}$ indicates that more interactions between the RTIL and glucose are formed than between the glucose molecules. This is in agreement with our excess spectra as particularly the [EMIM] $\left[\mathrm{EtSO}_{4}\right]$-glucose/water interactions are represented (Fig. 3). This finding results from the comparison of the Raman excess spectrum with the RTIL-water/D-glucose spectra. It becomes obvious from the excess spectrum that interactions between glucose molecules can be neglected since particularly peaks which can be assigned to RTIL-water/glucose interactions show up. However, based on the work of Yue et al. $(2014)^{25,26}$ who found an improved enantioseparation in the presence of [EMIM] [EtSO $\left.{ }_{4}\right]$, it can be assumed that enantioselective interactions between the RTIL and glucose occur. With the knowledge of the mentioned studies, we can conclude that displayed RTIL-water interactions are overestimated and particularly RTIL-glucose interactions occur in this quaternary mixture. To improve the evaluation

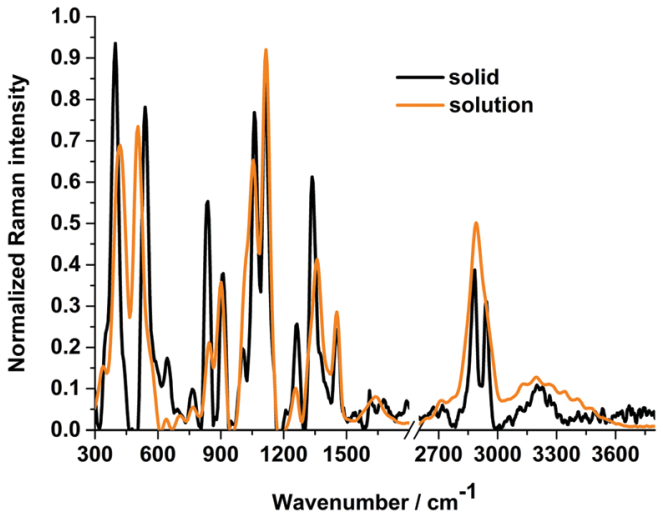

Fig. 4 Representation of the differences in the Raman signal of D-glucose obtained from the solid and aqueous phase (47 wt\%).

using excess calculations, a weighted Raman spectrum (eqn (4)) of the RTIL and water was subtracted based on the study of Carneiro et al. (2012). ${ }^{46}$ However, the results turned out to be still not meaningful enough as the influence of the glucose molecules cannot be considered in the excess calculations without introducing significant errors. Glucose is solid at room temperature whereas the other mixture components occur in the liquid phase. By using the Raman spectra of aqueous solutions we are not able to consider D-D- or L-L-glucose interactions as well as glucose-water interactions since we subtract the spectra of the aqueous glucose solutions from the spectra of the experimental mixture to receive the excess spectra. Using the spectra of the solid glucose we get problems since the Raman signal obtained from different phases significantly differ in the peak intensity, peak width and peak position (Fig. 4). This means we face a trade-off between an error in the peak position, peak width and intensity or in ignoring interactions.

Because of the problems associated with building up meaningful reference and thus excess spectra, we developed a model based on principal component analysis (PCA) to evaluate the position and the weight of changes in the intermolecular interaction structure of multi-component mixtures independent of the phase of the pure substance. This is due to the fact that PCA uses only the spectra of the mixtures while for excess spectroscopy the ideal spectra are calculated based on the spectra of the pure components. In Fig. 5, the loadings of the first principal component (PC1) are plotted versus the wavenumber. The corresponding molecular vibrations are assigned in Table 2. Since the loadings represent the largest signal variance, it is now possible to evaluate the significance of changes in the intermolecular interaction structure. For that, we compared the loadings of the first four principal components depending on the wavenumber with the Raman spectra of RTIL and glucose or water. As an example, Fig. 5 shows this approach for PC1. By that, it is possible to assign molecular vibrations influenced by RTIL-glucose or RTIL-water interactions. The explained variances of the first four principal components (PC1-4) are summarized in descending order in Table 1 supporting our results. These PCs explain $93.85 \%$ of the whole variances of all spectra, which is satisfactory for our approach. 


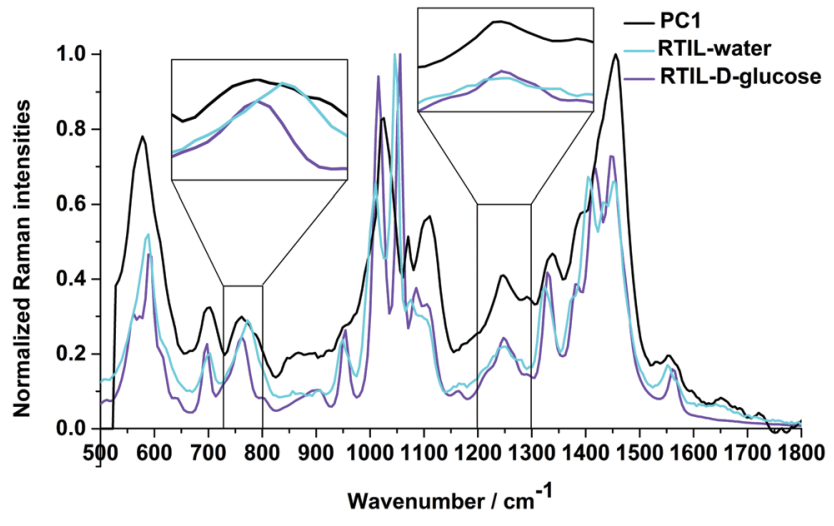

Fig. 5 Comparison of the loading of PC1 and the two Raman spectra of RTIL and glucose or water to display the weighted intermolecular interactions in the loading.

Table 1 Explained variances of the first four PCs

\begin{tabular}{lllll}
\hline Principal component & PC1 & PC2 & PC3 & PC4 \\
\hline Explained variance $/ \%$ & 73.80 & 11.94 & 5.35 & 2.76
\end{tabular}

Table 2 is used as an example for the PCs and shows significant weights for vibrational frequencies of [EMIM] $\left[\mathrm{EtSO}_{4}\right]$ and glucose. To receive normalized weightings the loadings are divided through their maximum. Therewith, it is possible to highlight peaks with strong variances in the spectra due to a weighted value of the peak near 1 . The vibrations weighted at 1025,1110 and $1333 \mathrm{~cm}^{-1}$ can be assigned to $\mathrm{COH}, \mathrm{CH}$ and $\mathrm{CH}_{2}$ vibrations of glucose, respectively. In addition, the $\delta\left(\mathrm{CH}_{2}\right)$-vibration of glucose shows a high weight. Thus, it can be assumed that particularly the $\mathrm{OH}$-group at the $\mathrm{C}_{6}$-atom of glucose forms hydrogen bonds with the ethyl sulphate anion of $[\mathrm{EMIM}]\left[\mathrm{EtSO}_{4}\right]$ since the $\delta\left(\mathrm{CH}_{2}\right)$-vibration is directly influenced by the formation of hydrogen bonds at the $\mathrm{OH}$-group of the $\mathrm{C}_{6}$-atom of glucose.

Moreover, based on Fig. 5 and Table 2 it can be concluded that the interactions between $[\mathrm{EMIM}]\left[\mathrm{EtSO}_{4}\right]$ and glucose dominate the overall interaction structure, whereas the intermolecular interactions between the RTIL and water can be neglected. This statement can be derived from the fact that the vibration of water centred at $1650 \mathrm{~cm}^{-1}$ shows only a normalized weighting of 0.08 , which indicates that these vibrations can be disregarded. From this observation it may also be concluded that the amount of water in the sample does not significantly influence the relevant interactions and therefore does not play a role in the evaluation.

Comparing PC2, PC3 and PC4 with the spectra of RTIL and water or D-glucose, respectively, we conclude that the RTILglucose interactions dominate in the quaternary mixture as they are represented in the first two PCs while RTIL-water interactions are first displayed in PC3 and PC4. This result underlines the significance of the PCA approach although the strengths of the interactions cannot be determined in detail. The conclusion on the strength of the interactions is in concert with the finding of an improved enantioseparation in the
Table 2 Assignment of the weighted peaks in the loading of $\mathrm{PC1}^{27,32,34,48,49}$

\begin{tabular}{|c|c|c|c|}
\hline $\begin{array}{l}\text { Wavenumber/ } \\
\mathrm{cm}^{-1}\end{array}$ & Vibration & Molecule & $\begin{array}{l}\text { Normalized } \\
\text { weighting/- }\end{array}$ \\
\hline 584 & $\nu(\mathrm{CN}), \omega(\mathrm{OSO})$ & {$[\mathrm{EMIM}]\left[\mathrm{EtSO}_{4}\right]$} & 0.76 \\
\hline 698 & $\omega(\mathrm{CH})$ & {$[\mathrm{EMIM}]\left[\mathrm{EtSO}_{4}\right]$} & 0.32 \\
\hline 772 & $\begin{array}{l}\delta(\mathrm{CH}), \gamma(\mathrm{CH}), \omega\left(\mathrm{CH}_{2}\right), \\
\omega(\mathrm{SO})\end{array}$ & {$[\mathrm{EMIM}]\left[\mathrm{EtSO}_{4}\right]$} & 0.29 \\
\hline 1025 & $\delta(\mathrm{COH})$ & Glucose & 0.83 \\
\hline 1076 & $\nu(\mathrm{COS}), \delta(\mathrm{CH}), \tau\left(\mathrm{CH}_{2}\right)$ & {$[\mathrm{EMIM}]\left[\mathrm{EtSO}_{4}\right]$} & 0.45 \\
\hline 1110 & $\delta(\mathrm{CH}), \delta(\mathrm{COH})$ & Glucose & 0.57 \\
\hline 1248 & $\tau\left(\mathrm{CH}_{2}\right), \omega\left(\mathrm{CH}_{2}\right)$ & {$[\mathrm{EMIM}]\left[\mathrm{EtSO}_{4}\right]$} & 0.41 \\
\hline 1333 & $\delta\left(\mathrm{CH}_{2}\right)$ & Glucose & 0.46 \\
\hline 1451 & $s\left(\mathrm{CH}_{3}\right), s\left(\mathrm{CH}_{2}\right), \delta_{\mathrm{as}}\left(\mathrm{CH}_{3}\right)$ & {$[\mathrm{EMIM}]\left[\mathrm{EtSO}_{4}\right]$} & 0.98 \\
\hline 1556 & $\begin{array}{l}r(\mathrm{CN}), \nu_{\text {ip,as }}(\operatorname{ring}), \nu\left(\mathrm{CH}_{3} \mathrm{~N}\right), \\
\nu\left(\mathrm{CH}_{2} \mathrm{~N}\right)\end{array}$ & {$[\mathrm{EMIM}]\left[\mathrm{EtSO}_{4}\right]$} & 0.20 \\
\hline 1655 & $\delta(\mathrm{OH})$ & Water & 0.08 \\
\hline
\end{tabular}

$\delta$ : in plane bending, $\gamma$ : out of plane bending, $\tau$ : twisting, $\rho$ : rocking, $\omega$ : wagging, $\sigma$ : scissoring, $\nu$ : stretching.

presence of imidazolium-based ionic liquids. This detailed information cannot be extracted by means of excess calculations. This example shows the power of principal component analysis to analyse multi-component systems by means of Raman spectroscopy.

The mixing ratio $r_{\mathrm{m}}$ is plotted against the scores of PC1 (Fig. 6) to further support our results. The scores represent the coordinate of the original spectrum in the factor space. Additionally, the scores plot in Fig. 6 shows the concentrationdependent changes in the intermolecular interaction structure, but do not offer a direct possibility of determining the enantiomer ratio. To that end, additional analysis methods would have to be applied such as partial least squares regression as a multivariate data analysis method. In the plot of Fig. 6, the scores of PC1 are shown as an average value from 10 individual spectra together with their respective standard variations. As it can be seen in Fig. 6 a small enantiomeric excess of $\mathrm{D}^{-}$or L-glucose $\left(r_{\mathrm{m}}=0.80\right.$ and 1.22) leads to a great shift in the value of the scores of PC1. As the racemate behaves differently to mixtures with an enantiomeric excess of D- or L-glucose, respectively, this result shows how PCA extracts significant changes of the intermolecular interaction structure. Therefore, the weighting of the variances which is displayed in the PCs, which are represented according to the explained variances in descending order (Table 1), is of great

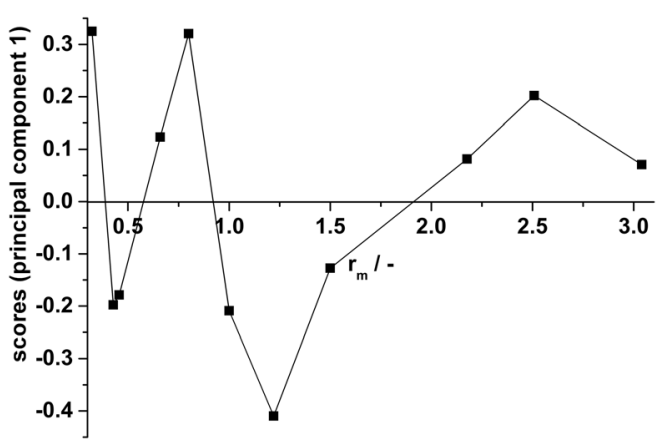

Fig. 6 Scores of PC1 (explained variance: $73.80 \%$ ) against the D-to-Lglucose ratios $r_{\mathrm{m}}$. 
importance. In summary, it has been shown that this type of analysis may be very useful for the investigation of intermolecular interactions between chiral molecules and RTILs. While in this study only one imidazolium-based ionic liquid has been exemplarily investigated, the approach might and should be applied to other RTILs.

\section{Conclusions}

The aim of this work was to compare the results of excess calculations and principal component analysis of Raman signals to probe and investigate the intermolecular interactions in multi-component mixtures. As a model system the interactions between D- and L-glucose, water, and [EMIM] $\left[\mathrm{EtSO}_{4}\right]$ were chosen. While straightforward analysis considering excess spectra did not lead to meaningful results, PCA revealed characteristic features of the mixtures. By comparing the loadings with the binary mixtures of the RTIL and glucose or water it became obvious that the weighting of the loadings reflects the occurring intermolecular interactions. The first and second principal components PC1 and PC2 strongly weight the RTIL-glucose interactions, while PC3 and PC4 exhibiting significantly lower explained variances weight RTIL-water as well as RTIL-glucose interactions. Moreover, the results of the PC1 analysis indicate that the main interaction is due to the formation of hydrogen bonds between the ethyl sulphate anion of [EMIM] $\left[\mathrm{EtSO}_{4}\right]$ and the $\mathrm{C}_{6}-\mathrm{OH}$-group of glucose. In addition, by analysing the scores of PC1 with regard to the mixing ratio the concentrationdependence of the interaction structure is obtained.

Moreover, in this study we are able to show that significant changes in the intermolecular interaction structure occur when a small enantiomeric excess exists. This can be highlighted by plotting the scores of PC1 versus $r_{\mathrm{m}}$. To expand this work for inline process analysis further studies regarding the analysis methods have to be performed. For the inline process control of enantiomeric separation processes a regression method, e.g. partial least squares regression (PLSR), is conceivable. In further studies the optimal regression method based on multivariate data analysis will be selected.

In conclusion, principal component analysis is a powerful tool for analysing multi-component mixtures in great detail regarding intermolecular interactions and their significance for the overall molecular structure and macroscopic behaviour. Besides, the results show that the combination of Raman spectroscopy and PCA or another multivariate data analysing tool offers the possibility of performing online process controls and optimizations in enantioseparation processes.

\section{Acknowledgements}

The authors gratefully acknowledge funding of the Erlangen Graduate School in Advanced Optical Technologies (SAOT) by the German Research Foundation (DFG) in the framework of the German excellence initiative. The authors also thank Lena Neumeier for her support in the lab.

\section{Notes and references}

1 B. Sharma, J. Xenobiot., 2014, 4, 14-19.

2 J. H. Lin and A. Y. H. Lu, Pharmacol. Rev., 1997, 49, 403-449.

3 J. McConathy and M. J. Owens, Primary Care Companion J. Clin. Psychiatry, 2003, 5, 70-73.

4 B. Kasprzyk-Hordern, Chem. Soc. Rev., 2010, 39, 4466-4503.

5 A. Rajendran, G. Paredes and M. Mazzotti, J. Chromatogr. A, 2009, 1216, 709-738.

6 M. Kaspereit, S. Swernath and A. Kienle, Org. Process Res. Dev., 2012, 16, 353-363.

7 S. Nimmig and M. Kaspereit, Chem. Eng. Process., 2013, 67, 89-98.

8 S. Robl, L. Gou, A. Gere, M. Sordo, H. Lorenz, A. Mayer, C. Pauls, K. Leonhard, A. Bardow, A. Seidel-Morgenstern and K. Schaber, Chem. Eng. Process., 2013, 67, 80-88.

9 G. Yang, J. Li, Y. Liu, T. L. Lowary and Y. Xu, Org. Biomol. Chem., 2010, 8, 3777-3783.

10 P. Heister, T. Lunskens, M. Thamer, A. Kartouzian, S. Gerlach, T. Verbiest and U. Heiz, Phys. Chem. Chem. Phys, 2014, 16, 7299-7306.

11 J. R. Avilés-Moreno, M. M. Quesada-Moreno, J. J. LópezGonzález and B. Martínez-Haya, J. Phys. Chem. B, 2013, 117, 9362-9370.

12 A. Ganesan, M. Brunger and F. Wang, Eur. Phys. J. D, 2013, 67, 1-12.

13 C. Johannessen, E. W. Blanch, C. Villani, S. Abbate, G. Longhi, N. R. Agarwal, M. Tommasini and D. A. Lightner, J. Phys. Chem. B, 2013, 117, 2221-2230.

14 N. Daugey, T. Brotin, N. Vanthuyne, D. Cavagnat and T. Buffeteau, J. Phys. Chem. B, 2014, 118, 5211-5217.

15 W. Li, J. Liang, W. Yang and J. Deng, ACS Appl. Mater. Interfaces, 2014, 6, 9790-9798.

16 Y. Wang, Z. Yu, W. Ji, Y. Tanaka, H. Sui, B. Zhao and Y. Ozaki, Angew. Chem., Int. Ed., 2014, 53, 13866-13870.

17 J. Kiefer, Analyst, 2015, 140, 5012-5018.

18 J. Kiefer and K. Noack, Analyst, 2015, 140, 1787-1790.

19 Ionic Liquids in Synthesis, ed. P. Wasserscheid and T. Welton, Wiley-VCH Verlag GmbH \& Co. KGaA, 2nd edn, 2008, DOI: 10.1002/9783527621194.

20 M. Matsumoto, K. Mochiduki, K. Fukunishi and K. Kondo, Sep. Purif. Technol., 2004, 40, 97-101.

21 M. L. Dietz, Sep. Sci. Technol., 2006, 41, 2047-2063.

22 U. Domańska and A. Rękawek, J. Solution Chem., 2009, 38, 739-751.

23 T. E. Sintra, R. Cruz, S. P. M. Ventura and J. A. P. Coutinho, J. Chem. Thermodyn., 2014, 77, 206-213.

24 Y. Tian, X. Meng and L. Shi, Fuel, 2014, 129, 225-230.

25 Y. Yue, X.-Y. Jiang, J.-G. Yu and K.-W. Tang, Chem. Pap., 2014, 68, 465-471.

26 Y. Yue, J. Yu, X. Jiang and Q. Deng, Curr. Anal. Chem., 2014, 10, 522-527.

27 J. Kiefer, J. Fries and A. Leipertz, Appl. Spectrosc., 2007, 61, 1306-1311.

28 U. Domańska and M. Laskowska, J. Solution Chem., 2008, 37, 1271-1287. 
29 L. E. Ficke, H. Rodríguez and J. F. Brennecke, J. Chem. Eng. Data, 2008, 53, 2112-2119.

30 M. Leskiv, C. E. S. Bernardes, M. E. Minas da Piedade and J. N. Canongia Lopes, J. Phys. Chem. B, 2010, 114, 13179-13188.

31 K. Noack, P. S. Schulz, N. Paape, J. Kiefer, P. Wasserscheid and A. Leipertz, Phys. Chem. Chem. Phys., 2010, 12, 14153-14161.

32 N. R. Dhumal, H. J. Kim and J. Kiefer, J. Phys. Chem. A, 2011, 115, 3551-3558.

33 J. Kiefer, M. M. Molina and K. Noack, ChemPhysChem, 2012, 13, 1213-1220.

34 K. Noack, A. Leipertz and J. Kiefer, J. Mol. Struct., 2012, 1018, 45-53.

35 H. Kise, K. Iwamoto and M. Sen, Bull. Chem. Soc. Jpn., 1982, 55, 3856-3860.

36 K. Kamogawa, K. Tajima, K. Hayakawa and T. Kitagawa, J. Phys. Chem., 1984, 88, 2494-2497.

37 K. Kamogawa and T. Kitagawa, J. Phys. Chem., 1985, 89, 1531-1537.

38 S. Wold, K. Esbensen and P. Geladi, Chemom. Intell. Lab. Syst., 1987, 2, 37-52.
39 P. R. Carey, J. Biol. Chem., 1999, 274, 26625-26628.

40 D. Ferrari, J. R. Diers, D. F. Bocian, N. C. Kaarsholm and M. F. Dunn, Biopolymers, 2001, 62, 249-260.

41 K. Bengraïne and T. F. Marhaba, J. Hazard. Mater., 2003, 100, 179-195.

42 C. Krafft, T. Knetschke, A. Siegner, R. H. W. Funk and R. Salzer, Vib. Spectrosc., 2003, 32, 75-83.

43 A. C. S. Talari, C. A. Evans, I. Holen, R. E. Coleman and I. U. Rehman, J. Raman Spectrosc., 2015, 46, 421-427.

44 K. Pearson, Philos. Mag., 1901, 2, 559-572.

45 H. Abdi and L. J. Williams, Wiley Interdiscip. Rev.: Comput. Stat., 2010, 2, 433-459.

46 A. P. Carneiro, O. Rodríguez and E. A. Macedo, Fluid Phase Equilib., 2012, 314, 22-28.

47 C. Molteni and M. Parrinello, J. Am. Chem. Soc., 1998, 120, 2168-2171.

48 P. D. Vasko, J. Blackwell and J. L. Koenig, Carbohydr. Res., 1972, 23, 407-416.

49 J. Grondin, J.-C. Lassègues, D. Cavagnat, T. Buffeteau, P. Johansson and R. Holomb, J. Raman Spectrosc., 2011, 42, 733-743. 